\title{
Numerical Solution of the One-Dimensional Heat Equation by Using Chebyshev Wavelets Method
}

\author{
HooshmandasI M. R*, Heydari M.H and Maalek Ghaini F.M
}

Faculty of Mathematics, Yazd University, Yazd, Iran

\begin{abstract}
In this paper, we develop an efficient Chebyshev wavelet method for well-known one-dimensional heat equation. In the proposed method we applied operational matrices of integration to get numerical solution of the onedimensional heat equation with Dirichlet boundary conditions. The power of this manageable method is confirmed. Moreover the use of Chebyshev wavelet is found to be accurate, simple and fast.
\end{abstract}

Keywords: Chebyshev wavelets; Operational matrix of integration; One-dimensional; Heat equation

\section{Introduction}

In this paper we study the physical problem of heat conduction in a rod of length L. This problem first studied by Fourier at the beginning of the 19th century in his celebrated volume on the analytical theory of heat, has become during the intervening century and a half the paradigm for the very extensive study of parabolic partial differential equations (PDEs), linear and nonlinear [1]. The temperature of a rod is governed by a PDE that is often defined by [2]:

$$
u_{t}=\bar{k} u_{x x}, \quad 0<x<L, \quad t \geq 0,
$$

where $u \equiv u(x, t)$ represents the temperature of the rod at the position $\mathrm{x}$ at time $\mathrm{t}$, and $\bar{k}$ is the thermal diffusivity of the material that measures the rod ability to heat conduction.

The domain of the solution is a semi-infinite strip of width $L$ that continues indefinitely in time. In a practical computation, the solution is obtained only for a finite time, say $T=t_{\max }$. Solution to equation 1 requires specification of initial condition at $t=0$ and boundary conditions at $\mathrm{x}=0$ and $\mathrm{x}=\mathrm{L}$. Simple initial and boundary conditions (IBCs) are:

$$
\begin{array}{lc}
u(x, 0)=f(x), & 0 \leq x \leq L, \\
u(0, t)=h_{1}(t), & t \geq 0 \\
u(L, t)=h_{2}(t), & t \geq 0
\end{array}
$$

The initial condition in equation 2 describes the initial temperature $\mathrm{u}$ at time $\mathrm{t}=0$ and the given boundary conditions in equation 3 and equation 4 indicate that the temperature of rod ends are functions of t. Other boundary conditions, e.g. gradient (Neumann) or mixed conditions, can be specified. In this article, only the conditions in equation 3 and 4 are considered. The existence and uniqueness property of this case problem have been studied by J. R. Cannon in [1]. It is of interest to note that the PDE in equation 1 arises in two different types, namely:

\section{Homogeneous heat equation}

$$
u_{t}=\bar{k} u_{x x}, \quad 0 \leq x \leq L, \quad t \geq 0
$$

Further, heat equation with a lateral heat loss is formally derived as a homogeneous PDE of the form:

$$
u_{t}=\bar{k} u_{x x}-c u, \quad 0 \leq x \leq L, \quad t \geq 0,
$$

where $\mathrm{c}$ is a positive constant.

\section{Inhomogeneous heat equation}

This type of equations is often given by

$$
u_{t}=\bar{k} u_{x x}+g(x, t), \quad 0 \leq x \leq L, \quad t \geq 0,
$$

where $\mathrm{g}(\mathrm{x} ; \mathrm{t})$ is called the heat source.

Heat equation mainly in one-dimension had been studied by many authors [3]. A comparative study between the traditional separation of variables method and Adomian method for heat equation had been examined by Gorguis and Chan [4]. Dehghan [5] considered the use of second-order finite difference scheme to solve the two-dimensional heat equation. After that, Mohebbi and Dehghan [6] presented a fourthorder compact finite difference approximation and cubic C1-spline collocation method for the solution with fourth-order accuracy in both space and time variables. Recently Dabral et al. [3], propose B-spline finite element method to get numerical solutions of one dimensional heat Equation.

Wavelet methods have been applied for solving PDEs from beginning of the early 1990s [7]. In the last two decades this problem has attracted great attention and numerous papers about this topic have been published. Due to this fact we must confine somewhat our analysis; in the following only PDEs of mathematical physics (elliptic, parabolic and hyperbolic equations) and of elastostatics are considered. From the first field of investigation the papers [8-13] can be cited. As to the elasticity problems we refer to the papers [14-20]. In all these papers different wavelet families have been applied. In most cases the wavelet coefficients were calculated by the Galerkin or collocation method, by it we have to evaluate integrals of some combinations of the wavelet functions (called also connection coefficients).

*Corresponding author: Hooshmandasl M R, Faculty of Mathematics, Yazd University, Yazd, Iran, E-mail: hooshmandasl@yazduni.ac.ir

Received September 25, 2012; Accepted December 08, 2012; Published December 11, 2012

Citation: HooshmandasI MR, Heydari MH, Maalek Ghaini FM (2012) Numerical Solution of the One-Dimensional Heat Equation by Using Chebyshev Wavelets Method. J Applied Computat Mathemat 1:122. doi:10.4172/2168-9679.1000122

Copyright: @ 2012 Hooshmandasl MR, et al. This is an open-access article distributed under the terms of the Creative Commons Attribution License, which permits unrestricted use, distribution, and reproduction in any medium, provided the original author and source are credited. 
The aim of the present work is to develop Chebyshev wavelet method with operational matrices of integration for solution the onedimensional heat equation with Dirichlet boundary conditions, which is fast, mathematically simple and guarantees the necessary accuracy for a relative small number of grid points. The outline of this article is as follows. In Properties of Chebyshev wavelets section; we describe properties of Chebyshev wavelet. In Description section; the proposed method is used to approximate the solution of the problem. After description section; the numerical examples of applying the method of this article are presented. Finally a conclusion is drawn in last section.

\section{Properties of Chebyshev Wavelets}

\section{Wavelets and Chebyshev wavelets}

Wavelets constitute a family of functions constructed from dilations and translations of a single function called the mother wavelet $\psi(t)$. When the dilation parameter $a$ and the translation parameter $b$ varies continuously we have the following family of continuous wavelets as [21]:

$$
\psi_{a, b}(t)=|a|^{-1 / 2} \psi\left(\frac{t-b}{a}\right), a, b \in \mathbb{R}, a \neq 0
$$

If we restrict the parameters $\mathrm{a}$ and $\mathrm{b}$ to discrete values as $a=a_{0}^{-k}, b=n b_{0} a_{0}^{-k}, a_{0}>1, b_{0}>0$ and $\mathrm{n}$ and $\mathrm{k}$ positive integers, we have the following family of discrete wavelets:

$$
\psi_{k, n}(t)=\left|a_{0}\right|^{k / 2} \psi\left(a_{0}^{k} t-n b_{0}\right)
$$

where $\psi_{k, n}(t)$ forms a wavelet basis for $L^{2}(\mathbb{R})$. In particular, when $\mathrm{a}_{0}=2$ and $\mathrm{b}_{0}=1, \psi_{k, n}(t)$ forms an orthonormal basis. That is $\left(\psi_{k, n}(t), \psi_{l, m}(t)\right)=\delta_{k l} \delta_{n m}$.

Chebyshev wavelets $\psi_{n, m}(t)=\psi(k, \hat{n}, m, t)$ have four arguments; $k \in \mathbb{N}, n=1,2, \ldots . .2^{k-1}$, and $\hat{n}=2 n-1$, moreover $\mathrm{m}$ is the degree of the Chebyshev polynomials of the first kind and $t$ is the normalized time. They are defined on the interval $[0,1]$ as:

$$
\psi_{n, m}(t)=\left\{\begin{array}{c}
2^{k / 2} \tilde{T}_{m}\left(2^{k} t-\hat{n}\right), \frac{\hat{n}-1}{2_{\text {otherwise }}^{k}} \leq t \leq \frac{\hat{n}}{2^{k}},
\end{array}\right.
$$

Where,

$$
\tilde{T}_{m}(t)=\left\{\begin{array}{c}
\frac{1}{\sqrt{\pi}}, \quad m=0 \\
\frac{\sqrt{\frac{2}{\pi} T_{m}(t),}}{m>0}
\end{array},\right.
$$

$\mathrm{m}=0 ; 1 ; \ldots ; \mathrm{M}-1$; and $\mathrm{M}$ is a fixed positive integer. The coefficients in (11) are used for orthonormality of the system. Here, $\left\{T_{m}(t), m \in \mathbb{N} \cup 0\right\}, \quad m=1,2,3 \ldots \ldots . \quad$ is the well-known Chebyshev polynomials of order $\mathrm{m}$ which are orthogonal with respect to the weight function $w(t)=1 / \sqrt{1-t^{2}}$ on the interval $[-1,1]$, and satisfy the following recursive formula:

$$
T_{0}(t)=1, T_{1}(t)=t \quad T_{m+1}(t)=2 t T_{m}(t)-T_{m-1}(T), \quad m=1,2,3 \ldots .
$$

We should note that in dealing with Chebyshev polynomials the weight function $\hat{w}(t)=w(2 t-1)$ have to be dilated and translated as

$$
w_{n}(t)=w\left(2_{t}^{k}-\hat{n}\right)
$$

to get orthogonal wavelets.

\section{Function approximation}

An arbitrary function $f(t) \in L^{2}(R)$ defined over [0;1] may be expanded into Chebyshev wavelets basis as

$$
f(t)=\sum_{p=1}^{\infty} \sum_{q=0}^{\infty} c_{p q} \psi_{p q}(t),
$$

where coefficients $c_{p q}=\left(f(t), \psi_{p q}(t)\right)$, in which (,) denotes the inner product. If the infinite series in (12) is truncated, then (12) can be written as

$$
f(t) \approx \sum_{p=1}^{2^{k-1}} \sum_{q=0}^{M-1} c_{p q} \psi_{p q}(t)=C^{T} \Psi(t),
$$

where $C$ and $\Psi(t)$ are $m \times 1\left(m=2^{k-1} M\right)$ matrices, given by

$$
\begin{gathered}
C=\left[\mathcal{C}_{10}, \mathcal{C}_{11}, \ldots, \mathcal{C}_{1 M-1}, \mathcal{C}_{20}, \ldots, \mathcal{C}_{2 M-1}, \ldots \mathcal{C}_{2^{k-1} 0}, \ldots, \mathcal{C}_{2^{k-1} M-1}\right]^{T}, \\
\Psi(t)=\left[\psi_{10}(t), \psi_{11}(t), \ldots, \psi_{1 M-1}(t), \psi_{20}(t), \ldots ., \psi_{2 M-1}(t), \ldots, \psi_{2^{k-1} 0}(t), \ldots . ., \psi_{2^{k-1} M-1}(t)\right]^{T}
\end{gathered}
$$

For simplicity, we write (13) as

$$
f(t) \approx \sum_{i=1}^{m} c_{i} \psi_{i}(t)=C^{T} \Psi(t),
$$

where $c_{i}=c_{p q}, \psi_{i}=\psi_{p q}$. The index $i$, is determined by the relation $i=M(p-1)+q+1$

Therefore we have

$$
\begin{aligned}
& C=\left[c_{1}, c_{2}, \ldots ., c_{m}\right]^{T}, \\
& \Psi(t)=\left[\psi_{1}(t), \psi_{2}(t), \ldots ., \psi_{m}(t)\right]^{T} .
\end{aligned}
$$

Similarly, an arbitrary function of two variables $u(x, t) \in L^{2}(\mathbb{R} \times \mathbb{R})$ defined over $[0,1) \times[0,1)$, may be expanded into Chebyshev wavelets basis as,

$$
u(x, t) \approx \sum_{i=1}^{m} \sum_{j=1}^{m} u_{i j} \psi_{i}(x) \psi_{j}(t)=\Psi^{T}(x) \mathrm{U} \Psi(t),
$$

where $\mathrm{U}=\left[\mathrm{u}_{\mathrm{ij}}\right]$ and $u_{i j}=\left(\psi_{i}(x),\left(u(x, t), \psi_{j}(t)\right)\right)$.

Taking the collocation points

$$
t_{i}=\frac{(2 i-1)}{2 m}, \quad i=1,2, \ldots . ., \hat{m},
$$

we define the $m \times m$ wavelet matrix $\Phi$ as

$$
\Phi=\left[\Psi\left(\frac{1}{2 m}\right), \Psi\left(\frac{3}{2 m}\right), \ldots, \Psi\left(\frac{2 m-1}{2 m}\right)\right] .
$$

\section{The operational matrices of Chebyshev wavelet}

Chen and Hsiao [22], introduced the concept of operational matrix in 1975, and Kilicman and Al Zhour [23] investigated the generalized integral operational matrix, that is, the integral of the matrix $\Psi(t)$ defined in (15) can be approximated as follows:

$$
\int_{0}^{t} \Psi(T) d T \cong P \Psi(t)
$$


where $\mathrm{P}$ is the $m \times m$ operational matrix of one-time integral of matrix $\Psi(t)$, similarly, we can compute the operational matrix $P^{n}$ of n-time integral of $\Psi(t)$. Wu and Hsiao [24] proposed a uniform method to obtain the corresponding integral operational matrix of different basis. For example, the operational matrix of $\Psi(t)$ can be expressed as:

$$
P=\Phi P_{B} \Phi^{-1},
$$

and in general the operational matrix $\mathrm{P}^{n}$ can be expressed as follows:

$$
P^{n}=\Phi P_{B}^{n} \Phi^{-1} \text {. }
$$

Here $P_{B}^{n}$ is the operational matrix of n-time integral of the Block Pulse Functions (BPF) [23]:

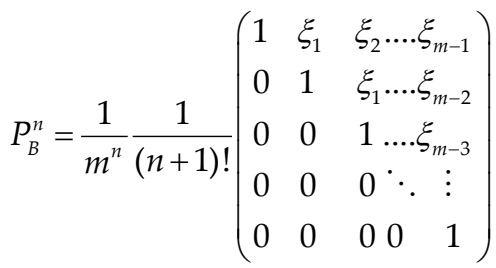

where, $\xi_{i}=(i+1)^{n+1}-2 i^{n+1}+(i-1)^{n+1}$.

\section{Description of Numerical Method}

In this section, we will use the Chebyshev wavelet operational matrices for solving the heat equation. Let us consider the inhomogeneous one-dimensional heat equation with lateral heat loss as:

$$
u_{t}=\bar{k} u_{x x}-c u+g(x, t), \quad 0 \leq x \leq 1, \quad 0 \leq t \leq 1 .
$$

And inhomogeneous Dirichlet boundary conditions:

$$
\begin{aligned}
& u(x, 0)=f(x), \\
& u(0, t)=h_{1}(t), \\
& u(1, t)=h_{2}(t),
\end{aligned}
$$

Where $f(x)$ and $h_{i}(t)$ are two times continuously differentiable functions on $[0,1]$ and $u(x, t) \in L^{2}(\mathbb{R})$. For solving this problem (2326), we assume:

$$
\frac{\partial^{3} u}{\partial t \partial x^{2}}=\Psi(x)^{T} \mathrm{U} \Psi(t)
$$

Where $\mathrm{U}=\left[\mathrm{u}_{i j}\right]_{m \times m}$ is an unknown matrix which should be found and $\Psi(t)$ is the vector that defined in (15). By integrating of (27) one time with respect to $t$ and considering (24) we obtain:

$$
\frac{\partial u}{\partial u}=\Psi(x)^{T}\left(P^{2}\right)^{T} \mathrm{U} \Psi(t)+\frac{\partial u}{\partial t} x=0+x \frac{\partial}{\partial x}\left(\frac{\partial u}{\partial t}\right) x=0 .
$$

Also by integrating of (27) two times with respect to $\mathrm{x}$ we get:

$$
\frac{\partial u}{\partial t}=\Psi(x)^{T}\left(P^{2}\right)^{T} \mathrm{U} \Psi(\mathrm{t})+\frac{\partial u}{\partial t}\left|x=0+x \frac{\partial}{\partial x}\left(\frac{\partial u}{\partial t}\right)\right| x=0 .
$$

By putting $\mathrm{x}=1$ into (29) and considering (25) and (26), we have:

$$
\frac{\partial u}{\partial t}=\Psi(x)^{T}\left(P^{2}\right) U \Psi(t)-x \Psi(1)^{T} U \Psi(t)+h_{1}^{\prime}(t)+x\left(h_{2}^{\prime}(t)-h_{1}^{\prime}(t)\right) .
$$

Now by integrating of (30) one times with respect to $t$ we get:

$$
u(x, t)=\Psi(x)^{T}\left(P^{2}\right)^{T} \mathrm{UP} \Psi(t)-x \Psi(1)^{T} \mathrm{UP} \Psi(t)+G(x, t),
$$

Where

$$
G(x, t)=f(x)+h_{1}(t)-h_{1}(0)+x\left(h_{2}(t)-h_{2}(0)-h_{1}(t)+h_{1}(0)\right) .
$$

Now by replacing (28), (30), and (31) into (23) we get:

$$
\begin{aligned}
& \Psi(x)^{T}\left[\left(P^{2}\right)^{T} \mathrm{U}-\bar{k} \mathrm{UP}+c\left(P^{2}\right)^{T} \mathrm{UP}\right] \Psi(t)-x \Psi(1)^{T}\left(P^{2}\right)^{T} \mathrm{U} \Psi(t)- \\
& \mathcal{c} x \Psi(1)^{T}\left(P^{2}\right)^{T} \mathrm{UP} \Psi(t) H(x, t),
\end{aligned}
$$

Where

$$
H(x, t)=h_{1}^{\prime}(t)+x\left(h_{2}^{\prime}(T)-h_{1}^{\prime}(T)\right)-\bar{k} f^{\prime \prime}(x)-c G(x, t)+g(x, t) .
$$

Equation (33) is a linear algebraic equation of $\mathrm{m}^{2}$ unknown variables $u_{i, j}(i, j=1,2, \ldots . ., m)$. Here, by taking collocation points, expressed in (17), for both $\mathrm{t}$ and $\mathrm{x}$ equation (33) is transformed into a linear system of algebraic equations. By solving this system and determined $\mathrm{U}$, we get the numerical solution of this problem by substitute $U$ into (31).

In this section, some numerical examples of the heat equation in form (23) with the initial and boundary conditions (24)-(26) with the proposed method are investigated. To show the efficiency of the present method, we report the root mean square error $\mathrm{L}_{2}$ and maximum error $L_{\infty}$ errors:

$$
\begin{aligned}
& L_{2}=\sqrt{\frac{1}{m} \sum_{i=1}^{m} \mid u\left(x_{i}, t_{i}\right)-\tilde{u}\left(x_{i},\left.t_{i}\right|^{2},\right.} \\
& L_{\infty}=\max _{1 \leq i \leq m}\left|u\left(x_{i}, t_{i}\right)-\tilde{u}\left(x_{i}, t_{i}\right)\right| .
\end{aligned}
$$

These examples are considered because both closed form solutions are available for them, and they have also been solved using other numerical schemes. All programs have been performed by Maple 14 and with 15 digits.

\section{Example 1}

In this example, we consider the classical heat equation in (23) with $\bar{k}=1, \mathrm{c}=0, \mathrm{~g}(\mathrm{x} ; \mathrm{t})=0$. The initial and boundary conditions are given by

$$
\begin{aligned}
& u(x, 0)=\sin (\pi x), \\
& u(0, t)=0, \quad 0 \leq t \leq T, \\
& u(1, t)=0 . \quad 0 \leq t \leq T .
\end{aligned}
$$

The exact solution of this problem is $u(x, t)=\sin (\pi x) e^{\pi^{2} t}$. The space-time graph of exact $(\mathrm{Ex})$ and numerical $(\mathrm{Nu})$ solutions for $\mathrm{m}=16$ $(\mathrm{M}=4 ; \mathrm{k}=3)$ are presented in figures 1 and 2 . The graph of exact and numerical solutions for some different times and $x \in[0,1 \mid$ are presented in figures 3 and 4 . The root-mean-square error $L_{2}$ and maximum error $L_{\infty}$ for $x \in|0,1|$ are presented in table 1 .

\section{Example 2}

Consider the heat equation (23) with $\mathrm{k}=1, \mathrm{c}=0$ and $\mathrm{g}(\mathrm{x} ; \mathrm{t})=0$. The initial and boundary conditions are given by $u(x, 0)=\mathrm{s} \sin (x), s$

$$
\begin{aligned}
& u(0, t)=0, \quad 0 \leq t \leq 1, \\
& u(1, t)=\sin (1) e^{-t}, \quad 0 \leq t \leq 1 .
\end{aligned}
$$

The exact solution of this problem is $u(x, t)=\sin (x) e^{-1}$ [2] 


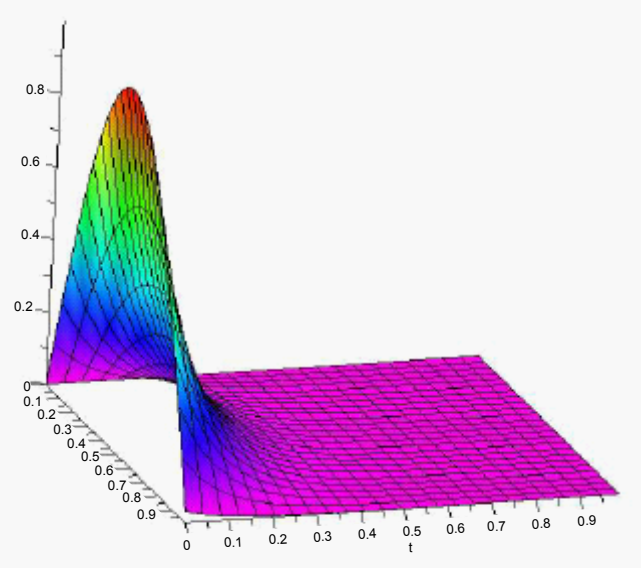

Figure 1: Ex solution for Example 1.

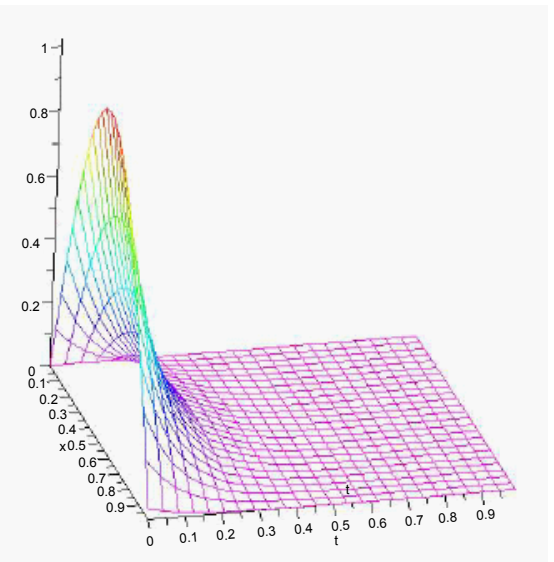

Figure 2: Nu solution for Example 1.

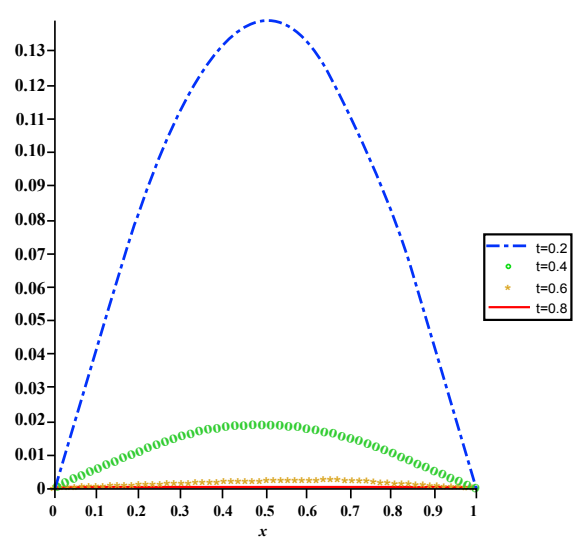

Figure 3: Ex solution in different values of $\mathrm{t}$.

\begin{tabular}{|l|l|l|l|l|l|l|}
\hline$t$ & $t=0.1$ & $t=0.3$ & $t=0.5$ & $t=0.7$ & $t=0.9$ & $t=1.0$ \\
\hline$L$ & $6.79 \times 10^{-3}$ & $3.76 \times 10^{-4}$ & $2.44 \times 10^{-4}$ & $3.17 \times 10^{-4}$ & $3.14 \times 10^{-3}$ & $3.32 \times 10^{-3}$ \\
\hline$L_{2}$ & $4.86 \times 10^{-3}$ & $8.87 \times 10^{-5}$ & $1.73 \times 10^{-3}$ & $2.04 \times 10^{-4}$ & $2.14 \times 10^{-3}$ & $2.15 \times 10^{-3}$ \\
\hline
\end{tabular}

Table 1: The $L_{1}$ and $L_{2}$ errors for some different values of $t$.

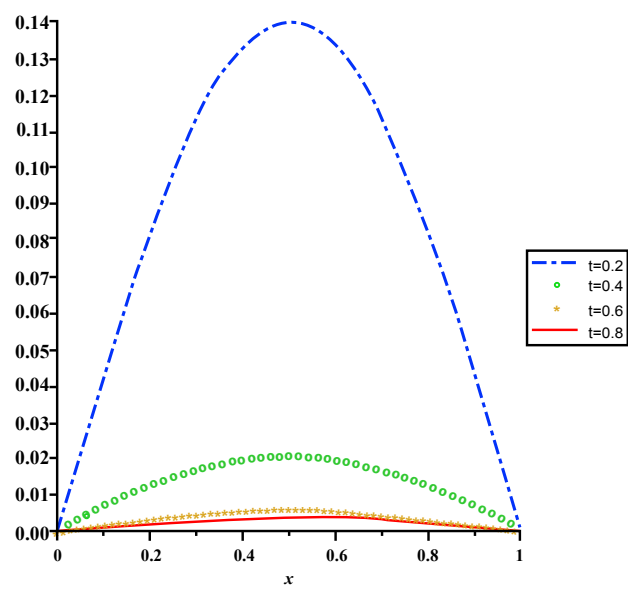

Figure 4: Nu solution in different values of $t$

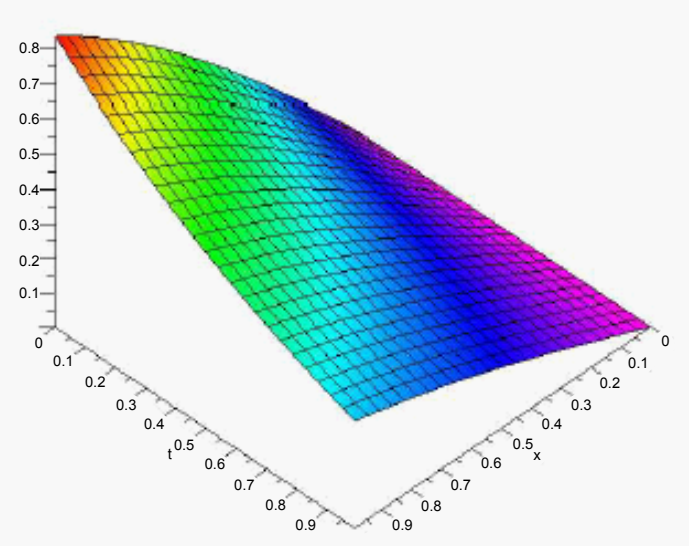

Figure 5: Ex solution for Example 2.

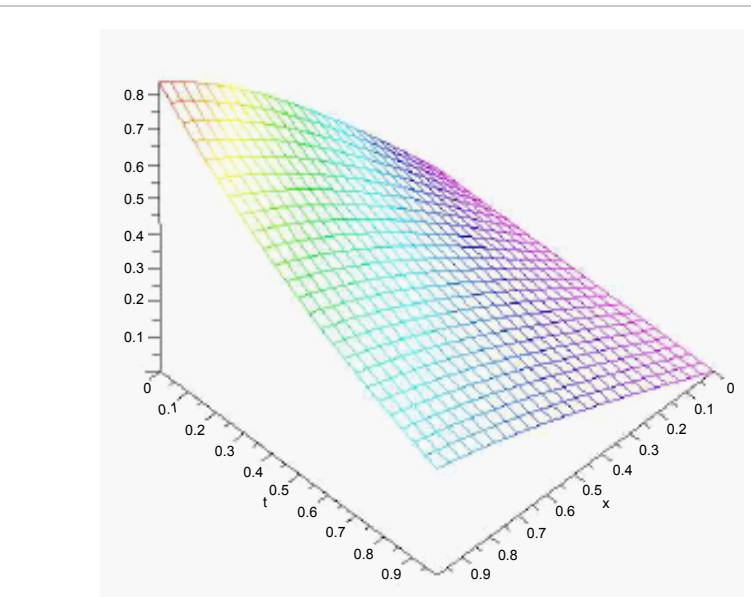

Figure 6: Nu solution for Example 2.

The space-time graph of exact and numerical solutions for $m=16$ are presented in figures 5 and 6 . The graph of exact and numerical solutions for some different times and $x \in[0,1]$ are presented in figures 7 and 8 . The 


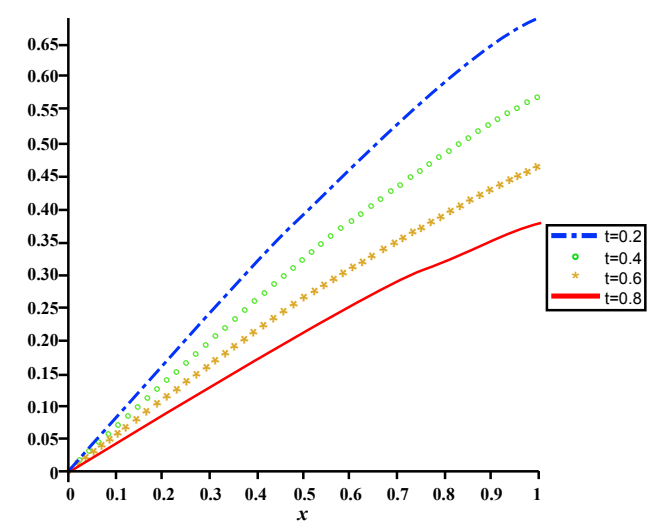

Figure 7: Ex solution in different values of $\mathrm{t}$

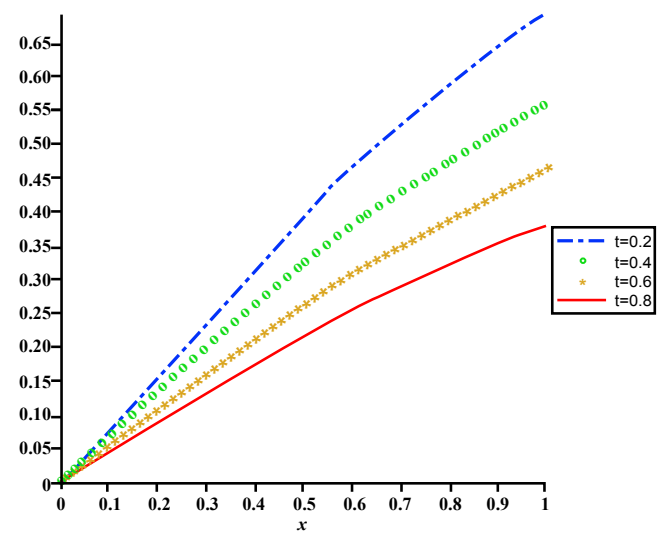

Figure 8: Nu solution in different values of $\mathrm{t}$.

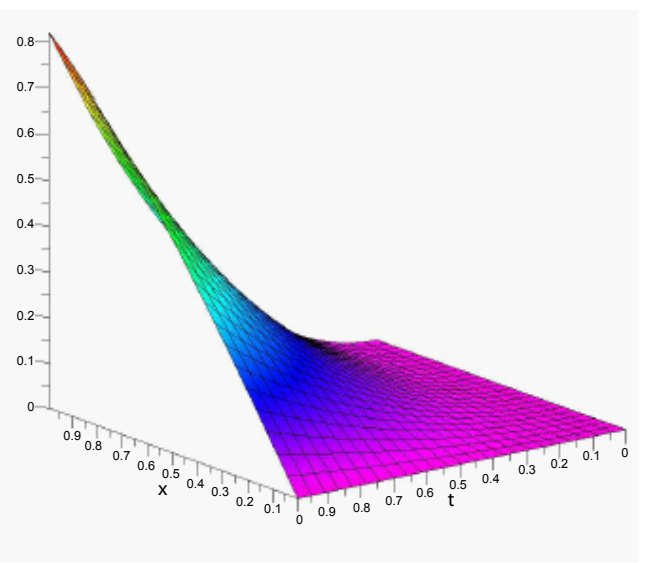

Figure 9: Ex solution for Example 3

\begin{tabular}{|l|l|l|l|l|l|l|}
\hline $\mathrm{t}$ & $\mathrm{t}=0.1$ & $\mathrm{t}=0.3$ & $\mathrm{t}=0.5$ & $\mathrm{t}=0.7$ & $\mathrm{t}=0.9$ & $\mathrm{t}=1.0$ \\
\hline$L_{\infty}$ & $1.07 \times 10^{-5}$ & $3.45 \times 10^{-6}$ & $5.13 \times 10^{-6}$ & $7.45 \times 10^{-6}$ & $9.47 \times 10^{-6}$ & $1.02 \times 10^{-5}$ \\
\hline $\mathrm{L}_{2}$ & $4.46 \times 10^{-6}$ & $2.15 \times 10^{-7}$ & $3.18 \times 10^{-6}$ & $4.71 \times 10^{-6}$ & $6.04 \times 10^{-6}$ & $6.55 \times 10^{-6}$ \\
\hline
\end{tabular}

Table 2: The $L_{\infty}$ and $L_{2}$ errors for some different values of $t$.

root-mean-square error $\mathrm{L}_{2}$ and maximum error $L_{\infty}$ for and $0 \leq x \leq 1$ are $0 \leq t \leq 1$ presented in table 2 .

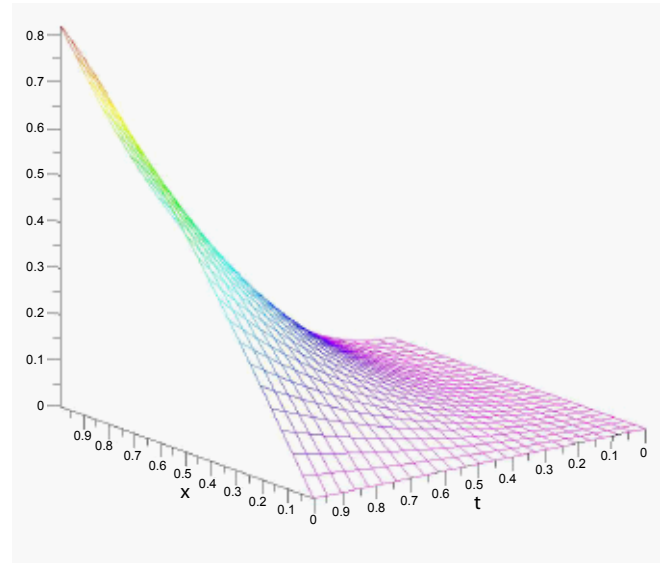

Figure 10: Nu solution for Example 3.

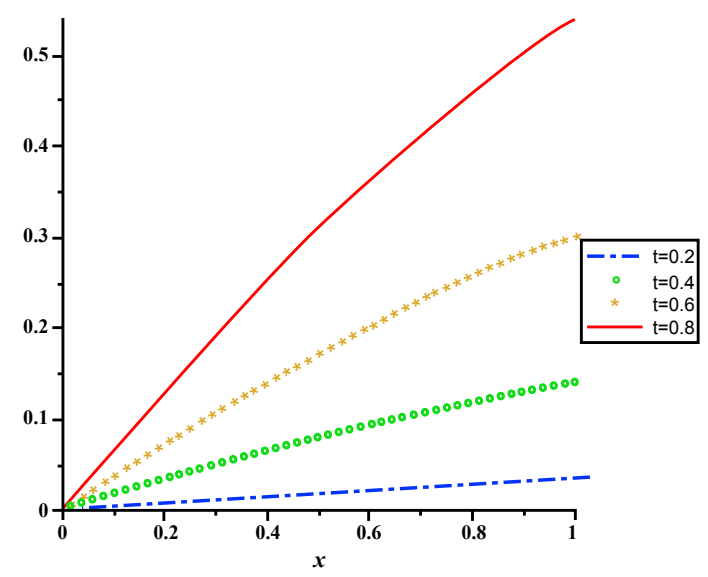

Figure 11: Ex solution in different values of $t$.

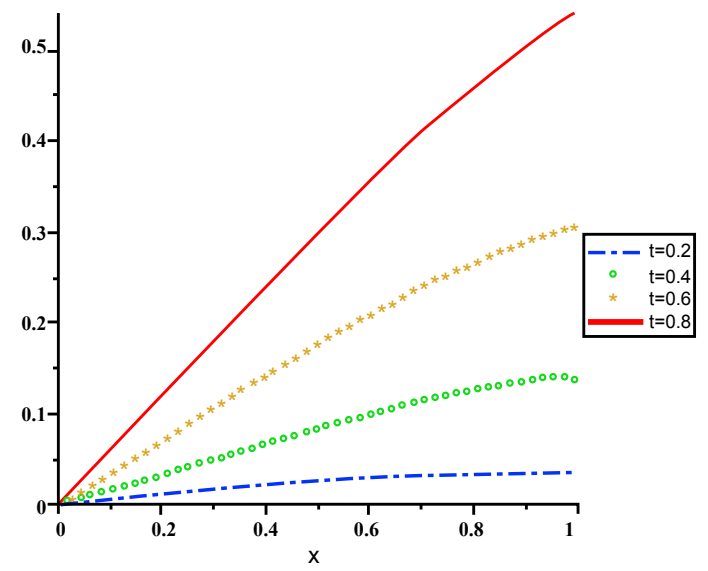

Figure 12: Nu solution in different values of $t$.

\section{Example 3}

In this example we consider the heat equation (23) with $\bar{k}=1, \mathrm{c}$ $=0$ and nonhomogeneous term $g(x, t)=\left(2 t+t^{2}\right) \sin (x)$ the initial and boundary conditions are given by 


$$
\begin{aligned}
& u(x, 0)=0, \\
& u(0, t)=0, \quad 0 \leq t \leq 1, \\
& u(1, t)=\sin (1) t^{2}, \quad 0 \leq t \leq 1
\end{aligned}
$$

The exact solution of this problem is $u(x, t)=t^{2} \sin (x)$ [2]. The space-time graph of exact and numerical solutions for $\mathrm{m}=16$ are presented in figures 9 and 10. The graph of exact and numerical solutions for some different times and $x \in[0,1]$ are presented in figures 11 and 12. The root-mean-square error $\mathrm{L}_{2}$ and maximum error $L_{\infty}$ for $0 \leq x \leq 1$ and $0 \leq t \leq 1$ are presented in table 3 .

\section{Example 4}

Finally consider the heat equation (23) with $\bar{k}=1$ and coefficient lateral heat $\mathrm{c}=2$ and $\mathrm{g}(\mathrm{x} ; \mathrm{t})=0$. The initial and boundary conditions are given by

$$
\begin{aligned}
& u(x, 0)=\sinh (x), \\
& u(0, t)=0, \quad 0 \leq t \leq 1, \\
& u(1, t)=\sinh (1) e^{-t}, \quad 0 \leq t \leq 1 .
\end{aligned}
$$

\begin{tabular}{|l|l|l|l|l|l|l|}
\hline $\mathrm{t}$ & $\mathrm{t}=0.1$ & $\mathrm{t}=0.3$ & $\mathrm{t}=0.5$ & $\mathrm{t}=0.7$ & $\mathrm{t}=0.9$ & $\mathrm{t}=1.0$ \\
\hline$L_{\infty}$ & $1.40 \times 10^{-3}$ & $5.78 \times 10^{-3}$ & $5.67 \times 10^{-3}$ & $1.10 \times 10^{-3}$ & $1.63 \times 10^{-3}$ & $2.27 \times 10^{-3}$ \\
\hline $\mathrm{L} 2$ & $1.23 \times 10^{-3}$ & $4.70 \times 10^{-3}$ & $4.89 \times 10^{-3}$ & $1.98 \times 10^{-3}$ & $1.11 \times 10^{-3}$ & $1.98 \times 10^{-3}$ \\
\hline
\end{tabular}

Table 3: The $L_{1}$ and $L_{2}$ errors for some different values of $t$.

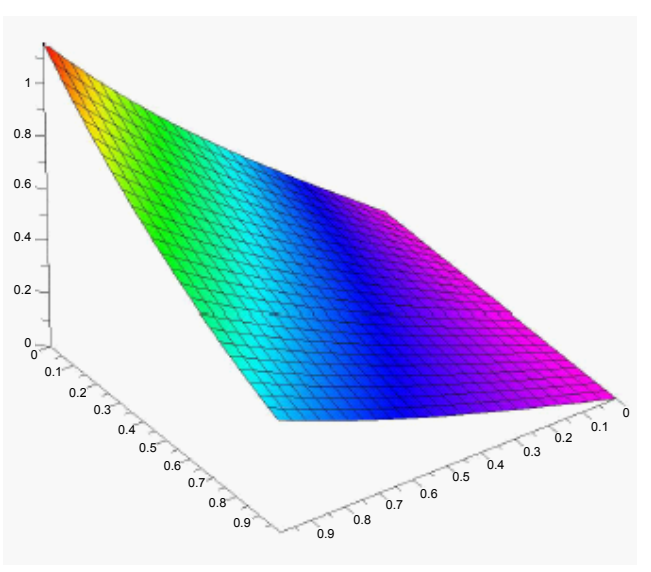

Figure 13: Ex solution for Example 3.

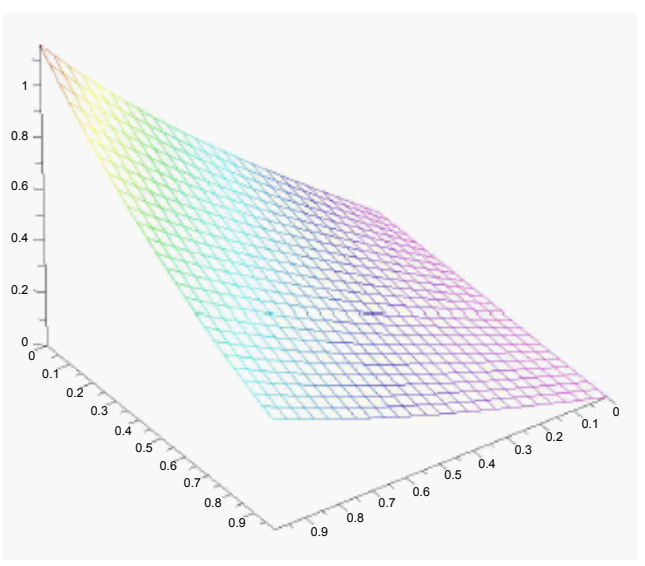

Figure 14: Nu solution for Example 3.

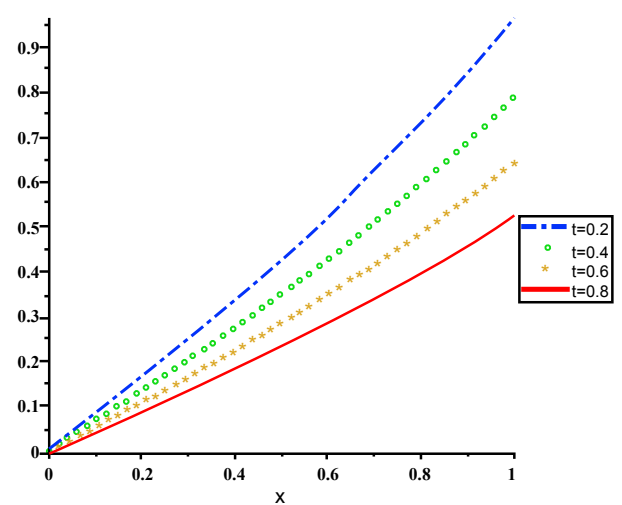

Figure 15: Ex solution in different values of $t$.

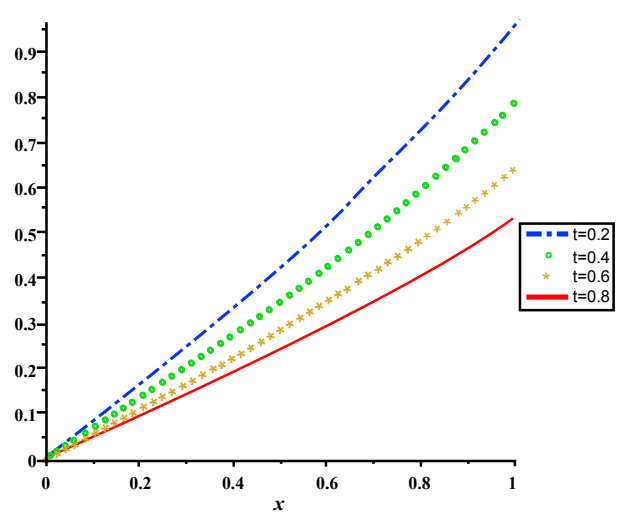

Figure 16: Nu solution in different values of $t$.

\begin{tabular}{|l|l|l|l|l|l|l|}
\hline $\mathrm{t}$ & $\mathrm{t}=0.1$ & $\mathrm{t}=0.3$ & $\mathrm{t}=0.5$ & $\mathrm{t}=0.7$ & $\mathrm{t}=0.9$ & $\mathrm{t}=1.0$ \\
\hline$L_{\infty}$ & $8.44 \times 10^{-3}$ & $8.10 \times 10^{-3}$ & $7.43 \times 10^{-3}$ & $9.18 \times 10^{-3}$ & $1.07 \times 10^{-3}$ & $1.15 \times 10^{-3}$ \\
\hline $\mathrm{L} 2$ & $6.01 \times 10^{-3}$ & $2.59 \times 10^{-3}$ & $4.78 \times 10^{-3}$ & $5.88 \times 10^{-3}$ & $6.89 \times 10^{-3}$ & $7.39 \times 10^{-3}$ \\
\hline
\end{tabular}

Table 4: The $L_{1}$ and $L_{2}$ errors for some different values of $t$.

The exact solution of this problem is $u(x, t)=e^{-t} \sinh (x)$ [2]. The space-time graph of exact and numerical solutions for $\mathrm{m}=16$ are presented in figures 13 and 14. The graph of exact and numerical solution for some different times and $x \in[0,1]$ are presented in figures 15 and 16. The root-mean-square error $\mathrm{L}_{2}$ and maximum error $L_{\infty}$ for $0 \leq x \leq 1$ and $0 \leq t \leq 1$ are presented in table 4 .

\section{Conclusion}

This paper presents a numerical method by combining wavelet function with operational matrices of integration to approximate numerical solutions of well-known one-dimensional heat equation. In the proposed method already a small number of grids points guarantee the necessary accuracy. The method is very convenient for solving boundary value problems, since the boundary condition are taken into account automatically. Also the proposed method is very simple in implementation and as the numerical results show the method is very efficient for numerical solution of mentioned problem and can be used for other partial differential equations.

\section{References}

1. Cannon JR (1984) The one dimensional heat equation. Washington. 
Citation: HooshmandasI MR, Heydari MH, Maalek Ghaini FM (2012) Numerical Solution of the One-Dimensional Heat Equation by Using Chebyshev Wavelets Method. J Applied Computat Mathemat 1:122. doi:10.4172/2168-9679.1000122

2. Wazwaz AM (2009) Partial differential equations and solitary waves theory. Springer, USA.

3. Dabral V, Kapoor S, Dhawan S (2011) Numerical simulation of one dimensional heat equation: B-spline finite element method. Indian Journal of Computer Science and Engineering 2: 222-235.

4. Gorguis A, Chan WKB (2008) Heat equation and its comparative solutions. Computers and Mathematics with Applications 55: 2973-2980.

5. M. Dehghan (2000) A finite difference method for a non-local boundary value problem for two-dimensional heat equation. Applied Mathematics and Computation 112: 133-142.

6. Mohebbi A, Dehghan M (2010) High-order compact solution of the onedimensional heat and advection-diffusion equations. Applied Mathematical Modelling 34: 3071-3084.

7. Lepik U (2011) Solving pdes with the aid of two-dimensional haar wavelets. Computers and Mathematics with Applications 61: 1873-1879.

8. Anderson U, Engquist B, Lredfelt G, Runborg O (1999) A contribution to wavelet-based subgrid modeling. Appl Comput Harmon Model 7: 151-164.

9. Cattani C (2004) Haar wavelets based technique in evolution problems. Chaos Proc Estonian Acad Sci Phys Math 1: 45-63.

10. Coult N (2006) Explicit formulas for wavelet-homogenized coefficients of elliptic operators. Appl Comput Harmon Anal 21: 360-375.

11. Chen X, Xiang J, Li B, He Z (2010) A study of multiscale wavelet-based elements for adaptive finite element analysis. Adv Eng Softw 41: 196-205.

12. Hariharan G, Kannan K, and Sharma KR (2009) Haar wavelet method for solving shers equation. Appl Math Comput 211: 284-292.

13. Mrazek P, Weickert J (2007) From two-dimensional nonlinear diffusion to coupled haar wavelet shrinkage. J Vis Commun Image Represent 18: 162-175.

14. Fan W, Qiao P (2009) A 2-d continuous wavelet transform of mode shape data for damage detection of plate structures. Internat J Solids Structures 46: 43794395.

15. Kim JE, Jang GW, Kim YY (2003) Adaptive multiscale wavelet-galerkin analysis for plane elasticity problems and its application to multiscale topology design optimization. Int J Solids Struct 46: 6473-6496.

16. Shen Y, Li W (2004) The natural integral equations of plane elasticity problem and its wavelet methods. Appl Math Comput 150: 417-438.

17. Chun Z, Zheng Z (2007) Three-dimensional analysis of functionally graded plate based on the haar wavelet method. Acta Mech Solida Sin 20: 95-102.

18. Lam HF, Ng CT (2008) A probabilistic method for the detection of obstructed cracks of beam-type structures using spatial wavelet transform. Probab Eng Mech 23: 237-245

19. Majak J, Pohlak M, Eerme M, Lepikult T (2009) Weak formulation based haar wavelet method for solving differential equations. Appl Math Comput 211: 488494.

20. Kajani MT, Vencheh AH, Ghasemi M (2009) The chebyshev wavelets operational matrix of integration and product operation matrix. Int $\mathrm{J}$ Comput Math 86: 1118-1125.

21. Chen CF, Hsiao CH (1975) Design of piecewise constant gains for optimal control via walsh functions. IEEE Transcations on Automatic control 20: 596603.

22. Kilicman A, Al Zhour ZA (2007) Kronecker operational matrices for fractional calculus and some applications. Appl Math Comput 187: 250-265.

23. Wu JL, Hsiao $\mathrm{CH}$ (1997) Haar wavelet method for solving lamped and distributed parameter systems. IEEE Proceeding-control Theory and Application 144: 87 94.

24. Tatari M, Dehghan M (2010) A method for solving partial differential equation via radial basis function: Application to the heat equation. Engineering analysis with Boundary Elements 34: 206-212. 